\title{
Implicaciones de la COVID-19 en la educación escolar; una revisión temprana de los artículos publicados en revistas académicas
}

\author{
Implications of COVID-19 in school education; an early review of \\ articles published in academic journals
}

Shamaly Alhelí Niño Carrascoํㅜ Juan Carlos Castellanos Ramírez² y Lissete Huerta Domínguez ${ }^{3}$

Fecha de recepción: 26 de agosto de 2020

Fecha de aceptación: 21 de noviembre de 2020

\footnotetext{
1- Nacionalidad: Mexicana. Grado: Doctorado en Psicología de la Educación. Adscripción: Universidad Autónoma de Baja California.Correo electrónico: shamaly.nino@uabc.edu.mx

2- Nacionalidad: Mexicana. Grado: Doctorado en Psicología de la Educación. Adscripción: Universidad Autónoma de Baja California.DORCID: https://orcid.org/0000-0002-0682-9085. Correo electrónico: juan.castellanos8@uabc.edu.mx 


\section{Resumen}

Este trabajo corresponde a un estudio de revisión sobre las obras publicadas en revistas académicas, en torno al impacto de la COVID-19 en la educación escolar. Mediante la técnica de análisis interjueces se revisaron 60 artículos publicados en revistas académicas de diferentes partes del mundo. Se constató que la producción de artículos recoge problemáticas educativas surgidas principalmente en Estados Unidos de América, India y China; esta producción se enmarca sustancialmente en las áreas de Tecnología educativa, Ciencias de la Educación y Ciencias de la Salud. También se observó que la gran mayoría de publicaciones corresponde a artículos de opinión que sitúan la reflexión en torno a la educación superior. En conjunto, los artículos emiten recomendaciones educativas de mejora en cinco niveles: políticas públicas en educación, atención y seguimiento institucional en el nivel educativo superior, atención y seguimiento curricular, procesos de enseñanza y de aprendizaje, y reconfiguración del sentido de la educación.

Palabras clave: COVID-19, desafíos educativos, educación escolar, políticas públicas, sistema educativo.

\section{Abstract}

This review study focuses on the works published in academic journals about the impact of COVID-19 on school education. Using the inter-judge analysis technique, 60 articles published in academic journals from different parts of the world were reviewed. It was found that the production of articles includes educational problems that arose mainly in the United States of America, India, and China; it is substantially framed in the areas of Educational Technology, Education Sciences and Health Sciences. It was also observed that the vast majority of publications correspond to opinion articles that place reflection at the higher educational level. Overall, the articles issue educational recommendations for improvement at five levels: public policies in education, care and monitoring of institutions at the higher educational level, care and monitoring of the curriculum, teaching and learning processes, and reconfiguration of the meaning of education.

Keywords: COVID-19, educational challenges, educational system, public policies, school education. 


\section{Introducción}

finales del año 2019 se reportaron 27 personas diagnosticadas con neumonía atípica en el Hospi-
tal Central de la Ciudad de Wuhan, China. El doctor Li Wenliang advirtió de manera rápida sobre
la presencia de un nuevo coronavirus de cepa desconocida (Petersen et al., 2020; Green, 2020; Parrish et al., 2020), sin embargo, fue hasta el 7 de enero de 2020 cuando el gobierno de la República Popular de China anunció, de manera oficial, un brote de coronavirus tipo 2 del síndrome respiratorio agudo grave, denominado SARS-CoV-2 (Zhang et al., 2020). Ante la capacidad de contagio y rápida propagación del nuevo virus, el 23 de enero se anunció el estado de cuarentena y cierre de todos los centros públicos en dicho país, entre ellos las escuelas de todos los niveles educativos (Peters et al., 2020; Prem et al., 2020).

En menos de tres meses, el virus se ha propagado exhibiendo algunas deficiencias en los sistemas de salud (Arango, 2020) y las limitaciones de los gobiernos locales para enfrentar las consecuencias financieras (McKee y Stuckler, 2020), sociales (Chakraborty y Maity, 2020) y educativas derivadas del confinamiento (Van y Parolin, 2020). El impacto global que ha causado el SARS-CoV-2 no tiene precedentes en la historia de la humanidad; lo que parecía ser solo un proverbio chino se ha convertido en una realidad demostrando que el leve aleteo de las alas de una mariposa se puede sentir al otro lado del mundo.

En lo que respecta a la educación formal, de acuerdo con Sun et al. (2020), los sistemas educativos de todo el mundo han experimentado grandes cambios durante los últimos meses. Por citar un ejemplo, los gobiernos, a través de los ministerios de educación, han impulsado políticas para asegurar el derecho a la educación, principalmente, a través del uso de plataformas tecnológicas para dar continuidad a las actividades académicas (Bozkurt y Sharma, 2020; Cóndor-Herrera, 2020; Holguín y Sandoval, 2020; Rogero-García, 2020; Yabin, 2020).

No obstante, se estima que la evolución de esta pandemia y su desenlace trasformará no solo el sistema escolar actual, sino también la prospectiva profesional de los educadores y la formación de nuevas generaciones de ciudadanos. Dichas transformaciones deben ser estudiadas desde un aparato reflexivo, crítico y científico que sirva de base para la elaboración de políticas educativas. En este contexto, es importante la creación de redes de colaboración entre académicos para compartir opiniones, experiencias e investigaciones en torno a las limitaciones, respuestas, desafíos y futuros escenarios del sistema educativo ante la emergencia sanitaria.

Con el propósito de presentar el estado del arte sobre el conocimiento teórico y empírico generado en torno a las implicaciones del SARS-Cov-2 en los sistemas educativos, se realizó un estudio documental en torno a 60 artículos publicados en revistas académicas de distintas partes del mundo. La producción analizada corresponde a artículos de investigación, intervención u opinión publicados dentro de la comunidad Combating the COVID-19 crisis together de la red social de investigadores ResearchGate.

\section{La lucha contra el SARS-CoV2: redes de colaboración y difusión del conocimiento}

La rápida propagación del virus SARS-CoV2 ha provocado grandes transformaciones en todos los sectores de la sociedad. Como lo manifestó el Director General de la Organización Mundial de la Salud 
(WHO, por sus siglas en inglés), más allá de una crisis mundial de salud pública, la pandemia también ha generado cambios en lo económico, laboral, educativo, psicológico, social y cultural; por lo tanto, esta lucha debe realizarse desde diversos frentes (World Health Organization [WHO], 2020). En este sentido, mientras que los médicos arriesgan su vida dentro de los hospitales, los economistas intentan dar estabilidad al mercado y las finanzas públicas, los psicólogos atienden la salud mental de la ciudadanía, los educadores se adaptan rápidamente a la enseñanza en línea para evitar la brecha en los aprendizajes de los estudiantes, entre otras muchas profesiones que hacen lo propio.

Otro frente amplio está constituido por investigadores y directores de revistas especializadas preocupados por difundir el conocimiento generado en torno al SARS-CoV2 (Brainard, 2020; Chan et al., 2020; Matias-Guiu; 2020). En situaciones normales, la publicación de artículos en revistas académicas suele tardar meses o años debido al proceso de evaluación rigurosa al que se someten (Björk y Solomon, 2013; Powell, 2016). Sin embargo, ante la emergencia sanitaria los directores de algunas revistas han suspendido la seriación de sus números habituales para priorizar la revisión de contribuciones relacionadas con el SARS-CoV2, mientras que otras han optado por abrir convocatorias de números monográficos para la evaluación y publicación de artículos en un tiempo récord. Algunas revistas han utilizado el sistema de publicación pre-print, que consiste en publicar los artículos antes de que se sometan a la revisión formal entre pares (Méndez, 2020).

Por su parte, las grandes empresas editoriales, como Elsevier, Taylor \& Francis, Wiley y Springer, han establecido redes de colaboración para brindar acceso gratuito a todas sus revistas asociadas. Incluso, en este esfuerzo por brindar rápido y fácil acceso a la producción científica, la red social de investigadores ResearchGate ha impulsado un proyecto denominado Combating the COVID-19 crisis together, en donde académicos de todo el mundo y de diferentes disciplinas comparten su producción reciente respecto a los efectos, retos y desafíos de la pandemia. A medida que la literatura incrementa, también se hace necesario realizar algunos cortes para dar cuenta del estado del conocimiento en cada una de las disciplinas y así poder reorientar las futuras líneas de trabajo relacionadas con la emergencia por el SARS-CoV2.

\subsection{El papel de las revistas académicas especializadas en el campo de la educación frente al SARS-CoV2}

La literatura sobre el SARS-CoV2 se ha disparado rápidamente dentro de las revistas especializadas (Carr, 2020); de acuerdo con el Instituto Allen para la Inteligencia Artificial (Allen Institute for AI, 2020), hasta finales de mayo se reportaban 140,000 artículos académicos. Aunque la mayor cantidad de artículos publicados hasta el momento corresponde al área de las Ciencias de la Salud, el esfuerzo realizado desde otros campos del conocimiento no ha sido menos importante.

Por ejemplo, en el campo de la Educación, la revista The Lancet publicó, el 07 de abril, un artículo de opinión elaborado por investigadores de Bélgica y Estados Unidos de América titulado COVID-19, school closures, and child poverty: a social crisis in the making (Van y Parolin, 2020). De manera similar, el 27 de abril la editorial Nature publicó, en una de sus revistas, un trabajo de investigación realizado en universidades chinas titulado Coronavirus pushes education online (Sun et al., 2020). 
Asimismo, revistas de todo el mundo especializadas en el campo de la Educación han realizado invitaciones para la publicación de trabajos relacionadas con la emergencia educativa frente al COVID-19, por ejemplo: Postdigit Sci Educ, Pedagogical Research, Asian Journal of Distance Education, Journal of Advances in Education Research, Higher Education, Journal of Research on Tecnology in Education, Revista Internacional de Educación para la Justicia Social, Revista de Sociología de la Educación, entre muchas otras.

En efecto, la producción y difusión de artículos en el campo de la Educación también ha crecido de manera exacerbada y seguirá aumentando hacia finales del año. Como se ve, las revistas electrónicas están jugando un papel importante en la difusión del conocimiento educativo en torno al SARS$\mathrm{CoV} 2$ ofreciendo información de primera fuente para conocer los escenarios de emergencia educativa en otras partes del mundo. Bajo este marco, en este trabajo se realiza una revisión sistemática de las primeras publicaciones con el propósito de identificar el tipo de producción, sus características, aportes e implicaciones para el sistema educativo.

\section{Metodología}

De acuerdo con el procedimiento sugerido por Cooper (1988), la revisión de la literatura se hizo en dos momentos diferentes. Primero, se realizó una búsqueda sobre las obras publicadas en la red social de investigadores ResearchGate; en concreto, la búsqueda se realizó en la comunidad Combating the COVID-19 crisis together que fue generada ad hoc por dicha red social "to stay up to date on the latest COVID-19 research as it happens, get help and support others, and contribute to the body of research the international community is using to combat the current crisis." (COVID-19 research community, 2020, párr. 1).

Aunque las revisiones en torno a un tema de estudio regularmente inician con una búsqueda de literatura en las principales bases de datos (por ejemplo, Web of Science, Scopus, Scielo, Redalyc, entre otras), se optó por consultar la comunidad descrita justamente por la facilidad y prontitud de acceso de las primeras publicaciones de distintas investigaciones en curso; de hecho, antes de consultar los trabajos publicados en la comunidad Combating the COVID-19 crisis together, se realizó una búsqueda previa en las bases de datos habituales de este tipo de estudios, pero los resultados de la búsqueda fueron mínimos e insuficientes para realizar un análisis acorde con los objetivos de esta revisión.

En virtud de lo anterior, con el fin de seleccionar la mayor cantidad de trabajos posibles en la comunidad Combating the COVID-19 crisis together, la búsqueda se realizó, primero, con los términos en español "educación", "enseñanza en línea", “emergencia educativa” y, después, con los términos en inglés "education", "remote teaching" y "educational emergency"; en ambos casos, los términos también se combinaron con “COVID-19" y "SARS-CoV-2". La búsqueda realizada el 10 de mayo de 2020 arrojó 76 resultados.

En segundo lugar, se procedió al escrutinio de todos los documentos considerando los siguientes criterios de inclusión y exclusión:

- trabajos que están publicados en revistas científicas o académicas, por lo que se excluyen los que aparecen publicados en blogs o páginas web;

- trabajos que, por su extensión y formato, pueden considerarse como artículos o artículos cortos, por lo que se excluyen las correspondencias, cartas al editor y libros o capítulos de libros; 
- trabajos que se centran en el impacto de la crisis sanitaria por la COVID-19 en cualquiera de las dimensiones del ámbito educativo, por lo que se excluyen los relacionados con otras temáticas.

De acuerdo con la aplicación de los criterios anteriores se descartaron 16 trabajos (ver Cuadro 1), por lo que únicamente 60 cumplieron con los criterios establecidos; en el siguiente enlace se detallan las referencias de cada uno de los trabajos revisados: https://drive.google.com/file/d/1SqmUSedmeWPCID xqazBQs05anntBmBx2/view?usp=sharing.

\section{Cuadro 1}

Número de trabajos descartados de la revisión según la aplicación de criterios

\begin{tabular}{cr}
\hline Criterio & N \\
\hline Trabajos publicados en blogs o páginas web & 3 \\
\hline Trabajos que son correspondencia, cartas al editor, libros o capítulos de libros & 11 \\
Trabajos que corresponden a una temática diferente o nada relacionada con el impacto de la \\
COVID-19 en el ámbito educativo & 2 \\
Total & 16
\end{tabular}

\subsection{Análisis de datos}

Para asegurar la fiabilidad de los resultados, el procedimiento de análisis se desarrolló en siete momentos diferentes con la participación de tres investigadores especialistas en el campo de la tecnología educativa quienes han abordado, en diferentes trabajos, el estudio de los procesos colaborativos mediados por ordenador y el uso de tecnologías como herramientas mediadoras para la enseñanza y el aprendizaje.

Primero, uno de los investigadores elaboró una propuesta inicial del protocolo de análisis para la revisión de la literatura considerando los títulos de los trabajos para el establecimiento de las primeras categorías; toda vez que los tres investigadores revisaron los resúmenes de los trabajos, de manera conjunta discutieron la pertinencia de la primera propuesta, logrando precisar las categorías iniciales y agregar nuevas categorías.

Segundo, con la versión mejorada del protocolo, los tres investigadores procedieron al escrutinio de 15 artículos; cada investigador revisó, por separado, cinco artículos. Tercero, tras la primera ronda de análisis, los investigadores se reunieron en línea para comentar el proceso de revisión, así como las dudas o inconvenientes que enfrentaron con la aplicación del protocolo; como resultado de esta reunión se matizaron los criterios de análisis considerando la propia naturaleza de los datos.

Cuarto, con la nueva versión del protocolo, los investigadores analizaron 30 artículos más; cada investigador asumió el análisis de 10 artículos. Quinto, tras la segunda ronda de análisis, los investigadores nuevamente se reunieron y dieron por cerrado el protocolo de análisis. 
Sexto, con la versión definitiva del protocolo se analizaron los 15 artículos restantes (cinco artículos por investigador) y se corroboraron los análisis anteriores para asegurar la correcta aplicación de los criterios. Finalmente, las codificaciones resultantes se sometieron a un procedimiento de acuerdo interjueces y se realizó el cálculo de fiabilidad (Kappa de Cohen) obteniendo un k=0,85.

\section{Resultados}

A continuación se presentan los resultados de la revisión de los 60 trabajos en dos grandes bloques. Primero se presentan los datos de naturaleza bibliométrica, es decir, los que han permitido caracterizar la literatura revisada en función del país al que remiten, el área de conocimiento, el nivel educativo en el que se centran, el tipo del artículo y el objeto de interés o de análisis de los artículos. Después se presentan los datos de naturaleza cualitativa que, en cierta medida, abren líneas de trabajo a corto, mediano y largo plazo, como las recomendaciones emitidas al cierre de los artículos revisados y los instrumentos utilizados en los trabajos de investigación y sus avances reportados.

\subsection{Características generales de la literatura}

El primer aspecto de la caracterización general se relaciona con los países a los que remite el contenido de los artículos analizados. Como se observa en el Cuadro 2, los países en los que se ha centrado la mayoría de los artículos relacionados con la COVID-19 y su impacto en la educación son Estados Unidos de América y la India, ambos con 10 artículos, y en menor medida China (ocho artículos). Por el contrario, los países con el menor número de producciones son Canadá, Ecuador y México (dos artículos); de manera similar, se encuentra un conjunto de 16 países que presentan solo una producción.

\section{Cuadro 2}

Número de artículos en función del país al que remite su contenido

$\begin{array}{cc}\text { País } & \text { Artículos } \\ \text { Estados Unidos de América } & 10 \\ \text { China } & 10 \\ \text { España } & 8 \\ \text { Brasil } & 4 \\ \text { Reino Unido } & 3 \\ \text { Canadá } & 3 \\ \text { Ecuador } & 2 \\ \text { México } & 2 \\ \text { Otros países con una sola contribución } & 2 \\ \text { Total } & 16\end{array}$


De acuerdo con el Cuadro 3, se han identificado cinco áreas o disciplinas de las que emergen los artículos analizados: i) Tecnología educativa, ii) Ciencias de la Educación, iii) Ciencias de la Salud, iv) Políticas públicas y v) Otras disciplinas. Como se ve, el área que presenta el mayor número de contribuciones es, justamente, Tecnología Educativa (17 artículos), que es hasta cierto punto esperado, ya que las tecnologías de la información y la comunicación (TIC) son las herramientas que han posibilitado en gran medida la continuidad académica ante la emergencia sanitaria; en el caso opuesto, del área de Políticas públicas solo se han generado seis contribuciones. Finalmente, se identificó un conjunto de cinco artículos que provienen de otras áreas.

\section{Cuadro 3}

Número de artículos según el área o disciplina

$\begin{array}{cc}\text { Área / Disciplina } & \text { Artículos } \\ \text { Tecnología Educativa } & 17 \\ \text { Ciencias de la Educación } & 16 \\ \text { Ciencias de la Salud } & 16 \\ \text { Políticas Públicas } & 6 \\ \text { Otras disciplinas } & 5 \\ \text { Total } & 60\end{array}$

Respecto al nivel educativo (ver Cuadro 4), se identificó que del total de los artículos analizados 39 centran su atención en la Educación Superior, mientras que 13 abordan situaciones, problemáticas o propuestas sobre el sistema educativo del país al que remiten y solamente ocho artículos centran su interés en opinar, intervenir o investigar sobre el nivel de educación básica. Sobre este punto llama la atención, primero, que no hay trabajos que remitan a la educación media superior -o su equivalente en otros países- y, además, que siendo el nivel básico el que cuenta con el mayor número de estudiantes y docentes a nivel mundial son pocos los artículos que centran su interés en dicho nivel educativo.

\section{Cuadro 4}

Número de artículos que centran su interés en cada nivel educativo

\begin{tabular}{cc} 
Nivel educativo & Artículos \\
\hline Educación básica & 8 \\
Educación superior & 39 \\
Sistema educativo & 13 \\
Total & 60
\end{tabular}

Sobre el tipo de artículos, se identificaron cuatro categorías diferentes según la finalidad explícita en los mismos trabajos: artículos de intervención en el aula, artículos de intervención institucional, artículos de opinión y artículos de investigación. De acuerdo con el Cuadro 5, la mayor cantidad de trabajos 
- Revista de Ciencias Sociales y Humanidades. ISSN-P: 0188-9834 ISSN-E:2395-8669.

publicados corresponde a los artículos de opinión (33 artículos); esta categoría se caracteriza porque los autores expresan sus puntos de vista respecto al impacto de la COVID-19 en cualquiera de los ámbitos educativos. Otro buen conjunto de trabajos corresponde a los artículos de investigación (20 artículos), en los que se informan los hallazgos o resultados de determinados proyectos de investigación relacionados con una problemática educativa específica en torno a la COVID-19.

\section{Cuadro 5}

Tipos de artículos publicados en función de su contenido

\begin{tabular}{|c|c|c|}
\hline Categoría & Descripción & Artículos \\
\hline $\begin{array}{l}\text { Intervención en } \\
\text { el aula }\end{array}$ & $\begin{array}{l}\text { Obras donde los autores comparten sus experiencias como docentes, respecto } \\
\text { a los recursos y estrategias implementadas para asegurar el desarrollo del curso } \\
\text { académico en la modalidad en línea. }\end{array}$ & 6 \\
\hline $\begin{array}{l}\text { Intervención insti- } \\
\text { tucional }\end{array}$ & $\begin{array}{l}\text { Obras donde los autores comparten propuestas y acciones institucionales } \\
\text { implementadas por los centros o instituciones educativas para responder a la } \\
\text { emergencia por la COVID-19 y asegurar la continuidad del ciclo escolar mediante } \\
\text { la modalidad en línea. }\end{array}$ & 2 \\
\hline Opinión & $\begin{array}{c}\text { Obras donde los autores, a partir de sus conocimientos previos en el campo de la } \\
\text { educación, expresan sentires, juicios, percepciones o reflexiones sobre la situa- } \\
\text { ción que atraviesa el sistema educativo en general y las instituciones educativas } \\
\text { en particular frente a la pandemia por la COVID-19. }\end{array}$ & 32 \\
\hline Investigación & $\begin{array}{l}\text { Obras donde los autores informan los hallazgos o resultados de un proyecto de } \\
\text { investigación en el que se aborda una problemática educativa específica en torno } \\
\text { a la COVID-19. }\end{array}$ & 20 \\
\hline
\end{tabular}

Por su parte, el menor número de trabajos corresponde a los trabajos de intervención en el aula (seis artículos) y de intervención institucional (dos artículos); mientras que los primeros remiten a las experiencias docentes sobre la implementación de estrategias o recursos para dar seguimiento al proceso formativo de los estudiantes en la modalidad en línea, los trabajos de intervención institucional recogen las propuestas implementadas por los centros educativos para responder a la emergencia sanitaria.

En cuanto al objeto de interés de los trabajos (ver Cuadro 6), se observa que la mayoría se ha centrado en analizar los desafíos de los sistemas educativos ante la emergencia por la COVID-19 (20 artículos), especialmente los trabajos de opinión. Otros aspectos que han sido objeto de análisis remiten, por un lado, al papel de la tecnología para transitar de una modalidad educativa presencial a un escenario educativo en línea (14 artículos) y, por otro lado, a las consecuencias del confinamiento y el impacto del cierre de escuelas sobre el aprendizaje de los estudiantes (12 artículos); este último aspecto es especialmente abordado por los trabajos de investigación. 


\section{Cuadro 6}

Objeto de interés o de análisis de los artículos

\begin{tabular}{|c|c|c|c|c|c|c|}
\hline & Estudiantes & Docentes & Institución & $\begin{array}{l}\text { Tecnología } \\
\text { educativa }\end{array}$ & $\begin{array}{l}\text { Disposiciones/ } \\
\text { comunicados }\end{array}$ & $\begin{array}{l}\text { Sistema } \\
\text { educativo }\end{array}$ \\
\hline $\begin{array}{l}\text { Intervención en } \\
\text { el aula }\end{array}$ & 0 & 2 & 0 & 4 & 0 & 0 \\
\hline $\begin{array}{l}\text { Intervención } \\
\text { institucional }\end{array}$ & 0 & 0 & 0 & 2 & 0 & 0 \\
\hline Opinión & 3 & 3 & 4 & 5 & 1 & 16 \\
\hline Investigación & 9 & 3 & 0 & 3 & 1 & 4 \\
\hline Total & 12 & 8 & 4 & 14 & 2 & 20 \\
\hline
\end{tabular}

Por otra parte, los aspectos analizados con menor frecuencia -pero no por ello menos importantes- conciernen a los desafíos del docente (ocho artículos) y los retos institucionales (cuatro artículos) ante la emergencia sanitaria, así como las disposiciones o comunicados emitidos para regular acciones educativas frente a la emergencia (dos artículos). Cabe destacar que la mayor diversidad de objetos de interés o de análisis abordada se observó en los artículos de opinión y de investigación.

\subsection{Características cualitativas de la literatura}

A continuación, para la caracterización cualitativa de la literatura se presentan, primero, las recomendaciones emitidas al cierre de los 60 artículos revisados y, después, los instrumentos utilizados solo en los 20 trabajos de investigación y sus avances reportados.

\subsubsection{Recomendaciones emitidas para la mejora de los procesos educativos}

Respecto a las recomendaciones planteadas al cierre de los trabajos, de acuerdo con la Figura 1, se han podido identificar hasta cinco niveles diferentes en los que estas centran su intención de mejora: i) políticas públicas en educación, ii) atención y seguimiento institucional en el nivel educativo superior, iii) atención y seguimiento curricular, iv) procesos de enseñanza y de aprendizaje y v) sentido de la educación. 
Figura 1

Niveles de las recomendaciones planteadas en los trabajos de Opinión e Investigació

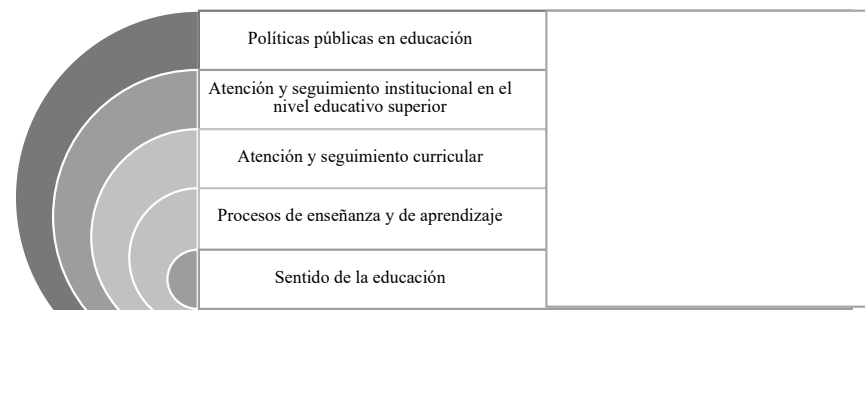

Sobre las recomendaciones del primer nivel -políticas públicas en educación- llama la atención que 20 de los 52 trabajos de Opinión e Investigación retoman esta urgencia por promover, desde un nivel más macro, acciones de fortalecimiento educativo que coadyuven a superar tanto las brechas de desigualdad social como las brechas tecnológicas con las que la mayoría de los sistemas educativos ha hecho frente a la emergencia sanitaria para dar continuidad a las actividades académicas.

Por citar un buen ejemplo, en el trabajo de Zhang y otros (2020) es posible identificar tres recomendaciones que, si bien impactan en otros niveles, requieren la formulación e implementación de políticas públicas como i) el promover políticas para priorizar el uso educativo de las herramientas tecnológicas en la sociedad, ii) el asegurar la implementación de programas de capacitación sistemática para profesores respecto al uso de las plataformas tecno-educativas y que exige, necesariamente, otorgar apoyo financiero, administrativo y legal a las instituciones educativas y iii) promover la investigación sobre la educación en línea para identificar los elementos necesarios para propiciar un aprendizaje de calidad en dichos ambientes educativos.

Aunque otros trabajos optan por enunciar solo una o dos recomendaciones, estas remarcan la obligación de los líderes de gobierno por atender con prontitud las deficiencias sociales, educativas y académicas que emergieron ante la COVID-19. Por ejemplo, los trabajos de Bozkurt y Sharma (2020), Cóndor-Herrera (2020) y Van y Parolin (2020) recomiendan que, ante los estragos económicos que ha causado la emergencia sanitaria, es urgente la formulación de políticas para apoyar monetariamente a los estudiantes de familias más vulnerables para, por un lado, disminuir las desigualdades económicas con las que enfrentarán el siguiente ciclo escolar $y$, por otro lado, superar las desigualdades tecnológicas en las que han estado inmersos.

En cuanto a las recomendaciones del segundo nivel -atención y seguimiento institucional en el nivel educativo superior-, los 18 trabajos identificados recomiendan que las diferentes instituciones educativas deben trabajar en la total implementación de la educación en línea, ya que esta permite la optimización de recursos, la organización de las actividades por parte de los docentes y, además, favorece la autonomía de los estudiantes en su aprendizaje. Por ejemplo, los trabajos de Bao (2020), Basilaia y Kvavadze (2020), Cáceres (2020) y Yabin (2020) coinciden en señalar la utilidad de la educación en línea en el período posterior a 
la pandemia, por lo que deben mejorarse las herramientas y plataformas digitales disponibles en las instituciones educativas y, a la vez, las metodologías de enseñanza para promover aprendizajes amplios y profundos en los estudiantes.

Dentro del mismo conjunto de trabajos, hay autores que además de plantear las recomendaciones anteriores hacen un llamado de atención a las instituciones para considerar otros aspectos de mejora igualmente importantes para el regreso a la nueva normalidad. Por ejemplo, mientras que algunos autores (Bozkurt y Sharma, 2020; Daniel, 2020; Van y Parolin, 2020) consideran que las instituciones deben realizar esfuerzos especiales para brindar seguimiento a los estudiantes más vulnerables bajo los principios de la educación para la emergencia, otros autores (Haletska et al., 2020; Peters et al., 2020; Satpathy y Ali, 2020; Shimil y Meenu, 2020) ponen especial interés en la formulación de políticas institucionales para el cuidado de la salud mental y la promoción de programas de rehabilitación para mitigar los efectos psicosociales de la emergencia sanitaria.

En las recomendaciones orientadas al tercer nivel -atención y seguimiento curricular- destacan siete trabajos que coinciden en el cambio obligatorio del curriculum de los distintos programas educativos considerando las transformaciones sociales, económicas y tecnológicas que se dieron a nivel mundial. Así, mientras Mirnaalini (2020), Mukhopadhyay et al. (2020), Pan (2020) y Shenoy et al. (2020) refieren que el aprendizaje en línea debe ser un contenido básico en los programas educativos y que, por lo tanto, se debe avanzar curricularmente hacia un sistema híbrido, Arora y Srinivasan (2020), Özer (2020) y Trujillo et al. (2020) coinciden en la premura por restablecer el vínculo entre el curriculum y las diferentes realidades de los estudiantes con el objetivo de que todos, sin excepción, puedan seguir aprendiendo.

Con respecto a las recomendaciones del cuarto nivel -procesos de enseñanza y de aprendizaje- se identificaron 12 trabajos dirigidos a la mejora del quehacer docente o bien de los estudiantes y sus procesos de aprendizaje. Sobre el quehacer docente, autores como Castaman y Rodrigues (2020) y Torres (2020) plantean que los profesores deben propiciar el aprendizaje significativo de los estudiantes otorgando pautas más cercanas a la cotidianidad, agregando comentarios personales en las retroalimentaciones de las actividades o, incluso, desarrollando propuestas instruccionales más centradas en los estudiantes y con alto grado de interacción entre ellos. Otros autores (Cáceres, 2020; Sun et al., 2020) destacan que el profesor puede ayudar a los estudiantes a reducir el estrés producido por el confinamiento y la propia contingencia sanitaria, por lo que este debe generar un clima de empatía y cuidado emocional entre los estudiantes para después enfocarse en los contenidos curriculares.

Sobre los estudiantes y sus procesos de aprendizaje, Leita et al. (2020) y Peters et al. (2020) coinciden al recomendar que los estudiantes también deben recibir formación sobre cómo regular su aprendizaje en línea y, junto con sus profesores, deben aprender a entender y regular sus propias emociones de cara al regreso a la nueva normalidad.

Finalmente, las recomendaciones del quinto nivel -sentido de la educación- son planteadas en 11 trabajos como una oportunidad de mejora para definir la nueva ruta de lo educativo. En este sentido, autores como Bozkurt y Sharma (2020), Mañero (2020), Murphy (2020), Mustafa (2020) y Thomas y Rogers (2020) coinciden en que se debe aprovechar el momento de inflexión para llevar a cabo un cambio de modelo pedagógico o instruccional, que no remita solo al cam- 
bio de una modalidad presencial a una modalidad en línea, sino también al cambio de modelos y prácticas de enseñanza; de hecho, Murphy (2020) advierte que, de no hacerlo así, se puede llegar a normalizar la enseñanza en línea de emergencia y, en consecuencia, esto llevaría a normalizar las brechas digitales y de aprendizaje.

\subsubsection{Instrumentos utilizados en los trabajos de investigación y avances reportados}

Como muestra el Cuadro 7, el análisis de los 20 trabajos de investigación ha permitido clasificarlos en función de tres rasgos: i) el método o instrumento utilizado para llevar a cabo el estudio, ii) el aspecto educativo analizado y iii) los resultados comunes más relevantes.

\section{Cuadro 7}

Instrumentos utilizados en los trabajos de investigación y avances reportados

\begin{tabular}{|c|c|c|c|}
\hline $\begin{array}{l}\text { Método o instrumen- } \\
\text { to utilizado }\end{array}$ & Aspecto educativo analizado & Resultados más relevantes & Referencia (2020) \\
\hline \multirow{2}{*}{$\begin{array}{l}\text { Cuestionarios o en- } \\
\text { cuestas de valoración } \\
\qquad N=13\end{array}$} & $\begin{array}{c}\text { Experiencias de los estudian- } \\
\text { tes o profesores sobre el uso } \\
\text { de las tecnologías en los cursos } \\
\text { en línea }\end{array}$ & $\begin{array}{l}\text { Los problemas de conectividad } \\
\text { y el desconocimiento de las he- } \\
\text { rramientas de las aulas virtuales } \\
\text { fueron algunos de los principales } \\
\text { desafíos vividos por estudiantes } \\
\text { y profesores }\end{array}$ & $\begin{array}{c}\text { Arora y Srinivasan } \\
\text { Castaman y Rodri- } \\
\text { gues } \\
\text { Grammegna } \\
\text { Shatakshi y Nardev } \\
\text { Sintema } \\
\text { Srivastava } \\
\text { Sun et al. } \\
\text { Trujillo et al. }\end{array}$ \\
\hline & $\begin{array}{c}\text { Experiencias emocionales, fac- } \\
\text { tores psicológicos o motivación } \\
\text { académica de los estudiantes } \\
\text { o profesores durante la emer- } \\
\text { gencia sanitaria }\end{array}$ & $\begin{array}{l}\text { El confinamiento impuso otras } \\
\text { actividades -de orden perso- } \\
\text { nal o familiar- que dificultaron } \\
\text { centrarse en las actividades } \\
\text { académicas } \\
\text { El compromiso de los estudian- } \\
\text { tes -o su motivación académica- } \\
\text { bajó en tiempos de COVID }\end{array}$ & $\begin{array}{l}\text { Haletska et al. } \\
\text { Mirnaalini } \\
\text { Pan } \\
\text { Satpathy y Ali } \\
\text { Shimil y Meenu }\end{array}$ \\
\hline $\begin{array}{l}\text { Registros de activi- } \\
\text { dad del Google Meet } \\
\qquad \mathrm{N}=1\end{array}$ & $\begin{array}{l}\text { Impacto de la implementación } \\
\text { del Google for Education y del } \\
\text { Google Meet en la transición a } \\
\text { la modalidad en línea }\end{array}$ & $\begin{array}{l}\text { Los profesores utilizaron inten- } \\
\text { samente el uso compartido de } \\
\text { la pantalla del escritorio para la } \\
\text { presentación de materiales }\end{array}$ & Basilaia y Kvavadze \\
\hline $\begin{array}{l}\text { No especifica } \\
\qquad N=1\end{array}$ & $\begin{array}{c}\text { Recursos o aplicaciones de } \\
\text { código abierto que favorezcan } \\
\text { la transición a la modalidad en } \\
\text { línea }\end{array}$ & $\begin{array}{l}\text { Se elaboró un catálogo de } \\
\text { aplicaciones de código abierto } \\
\text { para computadora y Android } \\
\text { que favorecen la continuidad } \\
\text { académica }\end{array}$ & $\begin{array}{l}\text { Oyeniran-Oluwashi- } \\
\text { na et al. }\end{array}$ \\
\hline $\begin{array}{l}\text { Entrevista } \\
\qquad \mathrm{N}=1\end{array}$ & $\begin{array}{c}\text { Experiencias de los profe- } \\
\text { sores sobre la transición a } \\
\text { la modalidad en línea y su } \\
\text { percepción sobre los procesos } \\
\text { de aprendizaje }\end{array}$ & $\begin{array}{l}\text { Desde la percepción de los } \\
\text { profesores, la modalidad en línea } \\
\text { mejoró el compromiso y la parti- } \\
\text { cipación de los estudiantes. }\end{array}$ & Shenoy et al. \\
\hline
\end{tabular}




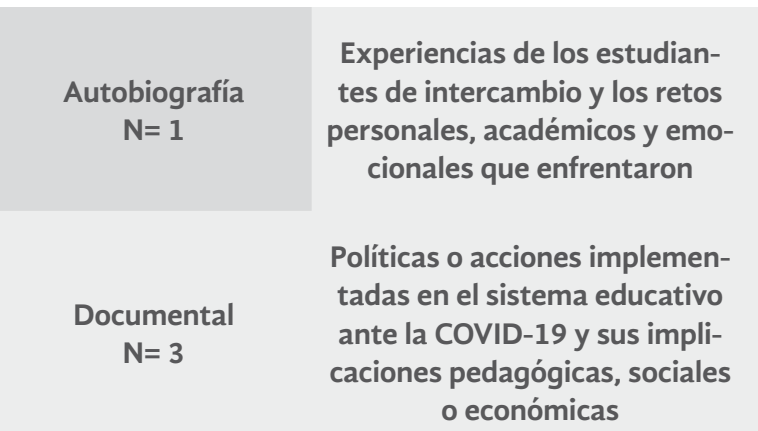

En algunos estudiantes se evidenció un desdén temprano por la educación en línea; en otros, las altas expectativas no fueron alcanzadas.

La existencia de la brecha social y la brecha digital dificultaron la implementación de la educación en línea y la continuidad académica
Peters et al.

Holguín y Sandoval Masuhay Mhlanga y Moloi

De acuerdo con la tabla anterior, es posible distinguir dos grupos de trabajos según el instrumento de recogida de datos empleado; mientras que un gran grupo opta por emplear cuestionarios o encuestas de valoración autoadministradas y los registros de actividad para recoger datos de naturaleza más cuantitativa (celdas sin sombrear), otro grupo prefiere el uso de entrevistas, autobiografías o del método documental para recoger datos de naturaleza más cualitativa (celdas sombreadas).

Dentro del primer grupo, los trabajos que emplearon cuestionarios o encuestas también se caracterizan por analizar dos aspectos educativos fundamentales; se centran en identificar las experiencias de los estudiantes o profesores sobre el uso de las tecnologías en los cursos en línea, por ejemplo Arora y Srinivasan (2020), Castaman y Rodrigues (2020) y Grammegna (2020), o bien sobre sus experiencias emocionales, factores psicológicos o motivación académica manifestados durante la emergencia sanitaria, como Haletska et al. (2020), Mirnaalini (2020) y Pan (2020). En cambio, en el trabajo de Basilaia y Kvavadze (2020), los registros de actividad se recogieron para analizar el impacto de una implementación tecnológica en la transición a la modalidad en línea.

En el segundo grupo, el interés común de tres trabajos es el análisis de las políticas o acciones implementadas por los líderes gubernamentales en el sistema educativo ante la COVID-19, así como sus implicaciones pedagógicas, sociales o económicas; para tal fin, Holguín y Sandoval (2020), Masuhay (2020) y Mhlanga y Moloi (2020) realizaron un análisis documental amplio de artículos en revistas de difusión, periódicos y revistas científicas. Por su parte, en el trabajo de Shenoy et al. (2020) se empleó la entrevista para recoger las experiencias de los profesores sobre el cambio de modalidad de enseñanza y su percepción sobre los procesos de aprendizaje de sus estudiantes, mientras que en el trabajo de Peters et al. (2020), las autobiografías fueron el instrumento mediante el que los estudiantes de intercambio (sujetos de estudio) hicieron constar sus experiencias, retos personales, académicos y emocionales vividos durante la contingencia sanitaria.

Por lo que respecta a los resultados comunes más relevantes, se evidencia que, en la mayoría de los casos, tanto estudiantes como profesores tuvieron sendos problemas al momento de transitar de una modalidad presencial a una modalidad en línea. Estos problemas fueron de carácter tecnológico (ver, por ejemplo, los trabajos de Shatakshi y Nardev, 2020; Sintema, 2020; Srivastava, 2020; Sun et al., 2020; Trujillo et al., 2020), de carácter psicológico o emocional (por ejemplo Satpathy y Ali, 2020; Shimil y Meenu, 2020) o incluso, en algunos casos, tuvieron que ver las propias expectativas de los profesores y estudiantes sobre la educación en línea (Shenoy et al., 2020; Peters et al., 2020).

Pese a que también se cuenta con resultados que evidencian un buen uso de determinadas aplicaciones de código abierto (Oyeniran-Oluwashina et al., 2020) o del impacto favorable de las plataformas 
digitales educativas para sostener la continuidad académica (Basilaia y Kvavadze, 2020), los resultados de los estudios con un espectro más macro (Holguín y Sandoval, 2020; Masuhay, 2020; Mhlanga y Moloi, 2020) evidencian que, efectivamente, la existencia de la brecha social y la brecha digital dificultaron la implementación de la educación en línea y la continuidad académica.

\section{Conclusión}

En este trabajo se presentó una revisión sistemática de los artículos publicados, hasta el día 10 de mayo de 2020, en torno a las implicaciones de la COVID-19 en la educación escolar. El propósito del estudio fue identificar el tipo de producción que se ha publicado, así como sus características, aportes e implicaciones para el sistema educativo. A partir de los resultados obtenidos, a continuación, se discuten los hallazgos de mayor relevancia y se plantean algunos retos para la comunidad académica, de cara a la realización de futuros trabajos.

En primer lugar, se destaca que las obras publicadas hasta el momento abordan problemáticas educativas surgidas, principalmente, en nueve países distintos (Estados Unidos de América, India, China, España, Brasil, Reino Unido, Canadá, Ecuador y México) y que gran parte de la producción se enmarca en las áreas de Tecnología educativa, Ciencias de la Educación y Ciencias de la Salud; sin embargo, prácticamente la mitad de los artículos revisados remiten a realidades educativas de Estados Unidos de América, India y China. Este resultado es consistente con los índices bibliométricos de producción mundial en los que, justamente, países como Estados Unidos de América y China aportan cerca del 30\% de la producción en revistas (Jiménez, 2016).

Por su parte, los escenarios educativos frente a la COVID-19 en países como Brasil, Ecuador y México han sido poco difundidos hasta el momento, a pesar del gran número de revistas educativas existentes en dichos países (Torres y Rovalo, 2015). En este sentido, es importante que las revistas educativas latinoamericanas consideren la posibilidad de abrir números monográficos o extraordinarios para recoger opiniones, experiencias y reportes de investigación sobre las circunstancias educativas vividas en dichos países durante la pandemia.

En segundo lugar, se resalta que la mayor cantidad de artículos publicados hasta el cierre de esta revisión remiten a las implicaciones de la COVID-19 en la educación superior, mientras que la literatura generada en torno a la educación básica es bastante reducida. De hecho, del total de los artículos analizados, solo ocho centran su interés en la educación básica a pesar de que, precisamente, la mayor cantidad de estudiantes afectados por la pandemia se concentra en el nivel básico; igualmente, llama la atención el hecho de que ninguno de los trabajos revisados se centre en el nivel medio superior. En este sentido, es de especial relevancia que los gobiernos impulsen políticas para la investigación y generación del conocimiento en dicho sector educativo, ya que ante una crisis educativa, de acuerdo con Murillo y Martínez (2019), la investigación es imprescindible para contribuir a la toma informada de decisiones.

En tercer lugar, respecto al tipo de obras publicadas en las revistas, se identificaron artículos de intervención en el aula, de intervención institucional, de opinión y de investigación. Hasta el momento, la mayor cantidad de trabajos publicados corresponde a los artículos de opinión, es 
decir, trabajos que plasman puntos de vista de expertos respecto al impacto de la COVID-19 en cualquiera de los ámbitos educativos. Aunque en menor medida, también se identificó un buen número de artículos de investigación, pero estos se desarrollan desde enfoques cuantitativos, a partir de la aplicación de cuestionarios o encuestas a estudiantes y profesores sobre sus experiencias y valoraciones en torno al uso de la tecnología para la educación remota de emergencia.

La predominancia de enfoques cuantitativos en las investigaciones puede estar relacionada con la inmediatez de los acontecimientos y la necesidad de contar con pronta información para la toma de decisiones; como manifiestan Rodero et al. (2016), la aplicación de cuestionarios es el método más sencillo y rápido que suele utilizarse para evaluar situaciones educativas, sin embargo, el solo hecho de elaborar una lista de preguntas y respuestas no garantiza la calidad de su medición.

Sería importante reforzar los trabajos de investigación a partir la realización de pruebas psicométricas para evaluar el grado de validez y fiabilidad de los instrumentos, ya que esto puede favorecer su aplicación en otros escenarios con características similares, así como la realización de estudios a gran escala. De manera complementaria, el uso de métodos cualitativos puede contribuir a la profundización de experiencias desde un sentir más individual y contextualizado de cada escenario reconociendo, de esta manera, las distintas realidades educativas frente a la pandemia.

En cuarto lugar, si bien los objetos de interés o de análisis de los distintos tipos de trabajos remiten fundamentalmente a los desafíos que están enfrentando los sistemas educativos ante la emergencia por la COVID-19, al papel que juega la tecnología en el tránsito de lo presencial a lo virtual y al impacto del cierre de escuelas en el aprendizaje de los estudiantes, es menester profundizar en los desafíos que enfrenta el docente ante situaciones de este tipo, es decir, sería importante indagar en sus necesidades de formación, en las inquietudes, miedos y retos que les impone el transitar de una enseñanza presencial a una de tipo virtual; sobre todo, se recalca la urgencia de diseñar procesos de formación (inicial y permanente) que realmente sean una herramienta con la que los docentes puedan hacer frente a estas vicisitudes.

Finalmente, en el conjunto de trabajos analizados también es posible identificar hasta cinco niveles diferentes de recomendaciones: i) políticas públicas en educación, ii) atención y seguimiento institucional en el nivel educativo superior, iii) atención y seguimiento curricular, iv) procesos de enseñanza y de aprendizaje y v) sentido de la educación. Sin duda, más allá de las características bibliométricas o cualitativas, todos los trabajos emiten recomendaciones para mejorar las prácticas educativas, en los distintos niveles, de cara a la nueva normalidad.

Lo anterior impone, a la vez, una serie de desafíos que han de atenderse a lo largo y ancho de los distintos niveles educativos; como bien señalan Bozkurt y Sharma (2020), Mañero (2020), Murphy (2020), Mustafa (2020) y Thomas y Rogers (2020), debe aprovecharse este momento de inflexión para buscar superar las brechas digitales y de aprendizaje que ha traído consigo la emergencia sanitaria (Murphy, 2020) y no solo eso; también se debe promover la implementación de medidas necesarias para garantizar el funcionamiento seguro de las instituciones educativas, la continuidad del aprendizaje, la atención de las situaciones de vulnerabilidad y, sobre todo, garantizar la protección y el bienestar de los estudiantes y docentes. 


\section{Referencias}

Allen Institute for AI. (2020, 23 de julio). COVID-19 Open Research Dataset Challenge (CORD-19). An AI challenge with AI2, CZI, MSR, Georgetown, NIH \& The White House. Kaggle. https://www.kaggle. com/allen-institute-for-ai/CORD-19-research-challenge

Arango, C. (2020). Lessons Learned From the Coronavirus Health Crisis in Madrid, Spain: How COVID-19 Has Changed Our Lives in the Last 2 Weeks. Biological psychiatry, 3223(20), 1-2. https:// doi.org/10.1016/j.biopsych.2020.04.003

Arora, A., y Srinivasan, R. (2020). Impact of Pandemic COVID-19 on the Teaching - Learning Process: A Study of Higher Education Teachers. Prabandhan: Indian Journal of Management, 13(4). http:// doi.org/10.17010/pijom\%2F2020\%2Fv13i4\%2F151825

Bao, W. (2020). COVID-19 and online teaching in higher education: A case study of Peking University. Human Behavior and Emerging Technologies, 2(2), 113-115. https://doi.org/10.1002/hbe2.191

Basilaia, G., y Kvavadze, D. (2020). Transition to Online Education in Schools during a SARS-CoV-2 Coronavirus (COVID-19) Pandemic in Georgia. Pedagogical Research, 5(4), 1-9. https://doi. org $/ 10.29333 / \mathrm{pr} / 7937$

Björk, B., y Solomon, D. (2013). The publishing delay in scholarly peer-reviewed journals. Journal of Informetrics, 7(4), 914-923. https://doi.org/10.1016/j.joi.2013.09.001

Bozkurt, A., y Sharma, R. (2020). Emergency remote teaching in a time of global crisis due to CoronaVirus pandemic. Asian Journal of Distance Education, 15(1), 1-6. https://doi.org/10.5281/zenodo.3778083

Brainard, J. (2020). Scientists are drowning in COVID-19 papers. Can new tools keep them afloat? Science. https://doi.org/10.1126/science.abc7839

Cáceres, K. (2020). Educación virtual: Creando espacios afectivos, de convivencia y aprendizaje en tiempos de COVID-19. CienciAmérica, 9(2), 38-44. http://doi.org/10.33210/ca.v9i2.284.

Carr, D. (2020, 31 de enero). Sharing research data and findings relevant to the novel coronavirus (COVID-19) outbreak. Wellcome. https://wellcome.ac.uk/coronavirus-covid-19/open-data

Castaman, A., y Rodrigues, R. (2020). Distance Education in the COVID crisis - 19: an experience report. Research, Society and Development, 9(6), 1-26. https://doi.org/10.33448/rsd-v9i6.3699

Chakraborty, I., y Maity, P. (2020). COVID-19 outbreak: Migration, effects on society, global environment and prevention. The Science of the total environment, 728, 1-7, https://doi.org/10.1016/j.scitotenv.2020.138882

Chan, A., Nickson, C., Rudolph, J., Lee, A., y Joynt, G. M. (2020, 30 de marzo). Social media for rapid knowledge dissemination: early experience from the COVID-19 pandemic. Anaesthesia. https://doi. org/10.1111/anae.15057

Cóndor-Herrera, O. (2020). Educar en tiempos de COVID. CienciAmérica, 9(2), 31-37. http://doi. org/10.33210/ca.v9i2.281

Cooper, H. M. (1988). Organizing knowledge syntheses: A taxonomy of literature reviews. Knowledge in Society, 1(1), 104-126. https://doi.org/10.1007/BF03177550

COVID-19 research community. (2020, 10 de mayo). Combating the COVID-19 crisis together. ResearchGate. https://www.researchgate.net/community/COVID-19 
Daniel, S. (2020, 20 de abril). Education and the COVID-19 pandemic. Prospects, Comparative Journal of Curriculum, Learning, and Assessment/ Springer. https://doi.org/10.1007/s11125-020-09464-3

Grammegna, A. (2020). The New Challenge of Education Value and Limits of Distance Learning Today. Journal of Education and Culture Studies, 4(2), 59-66. https://doi.org/10.22158/jecs.v4n2p59

Green, A. (2020). Obituary Li Wenliang. The Lancet, 395(10225), 682. https://doi.org/10.1016/S01406736(20)30382-2

Haletska, I., Klymanska, L., Klimanska, M., y Horoshenko, M. (2020). Students' emotional experience and behaviour during covid-19 quarantine: does fear or intrinsic motivation determine preventive behaviour? Psychological journal, 6(4), 35-52. https://doi.org/10.31108/1.2020.6.4.3

Holguín, E., y Sandoval, P. (2020). Resistir la Covid-19. Intersecciones en la Educación de Ciudad Juárez, México. Revista Internacional de Educación para la Justicia Social, 9(3), 7-23. https://doi. org/10.15366/riejs2020.9.3.001

Jiménez, N. (2016). Producción científica internacional en Educación Matemática. Estudio bibliométrico (1983-2012) [tesis de doctorado, Universidad de Córdoba, España].

Repositorio Institucional UCO. http://hdl.handle.net/10396/13759

Leita, A., Lopes, F., Franca, H., y Moreira, R. (2020). Tutoring in distance education in times of covid-19: relevant guidelines. Research, Society and Development journal, 9(5), 1-12. http://doi. org/10.33448/rsd-v9i5.3151

Mañero, J. (2020, 5 de mayo). Postdigital Brave New World and Its Educational Implications. Postdigital Science and Education. https://doi.org/10.1007/s42438-020-00129-0

Masuhay, E. (2020). Los adversarios en Covid-19 presentan un argumento hacia adelante en el esfuerzo educativo: resultando en desarrollar un concepto modular en el proceso de aprendizaje. American International Journal of Social Science Research, 5(2), 26-37. https://doi.org/10.46281/aijssr.v5i2.520

Matias-Guiu, J. (2020). The role of scientific journal editors during the COVID-19 pandemic. Neurología (English Edition), 35(4), 223-225. https://doi.org/10.1016/j.nrleng.2020.05.011

McKee, M., y Stuckler, D. (2020). If the world fails to protect the economy, COVID-19 will damage health not just now but also in the future. Nature Medicine, 26, 640-642. https://doi.org/10.1038/ s41591-020-0863-y

Mendez, J. (2020, 11 de abril). Compartir la ciencia en tiempos de coronavirus, una prueba de fuego para el sistema. Agencia SINC. https://www.agenciasinc.es/Reportajes/Compartir-la-ciencia-en-tiemposde-coronavirus-una-prueba-de-fuego-para-el-sistema

Mhlanga, D., y Moloi, T. (2020). COVID-19 and the Digital Transformation of Education: What We Are Learning in South Africa. Education sciences, 10(7), 1-11. https://doi.org/10.3390/educsci10070180

Mirnaalini, V. (2020). Determining the effect of motivation on computer mediated communication during COVID-19. International Journal of Indian Psychology, 8(2), 86-92. http://ijip.in/articles/ determining-the-effect-of-motivation-on-computer-mediated-communication-during-covid-19/

Mukhopadhyay, S., Booth, A., Calkins, S., Doxtader, E., Fine, S., Gardner, J., Gonzalez, R., Mirza, K., y Jiang, X. (2020, 4 de mayo). Leveraging Technology for Remote Learning in the Era of COVID-19 and Social Distancing: Tips and Resources for Pathology Educators and Trainees. Archives of Pathology \& Laboratory Medicine. https://doi.org/10.5858/arpa.2020-0201-ED 
Murillo, F., y Martínez, C. (2019). Una mirada a la investigación educativa en América Latina a partir de sus artículos. Revista Iberoamericana sobre Calidad, Eficacia y Cambio en Educación, 17(2), 5-25. https://doi.org/10.15366/reice2019.17.2.001

Murphy, M. (2020). COVID-19 and emergency eLearning: Consequences of the securitization of higher education for post-pandemic pedagogy. Contemporary Security Policy, 41(3), 492-505. https://oi. org/10.1080/13523260.2020.1761749

Mustafa, N. (2020). Impact of the 2019-2020 coronavirus pandemic on education. International Journal of Health Preferences Research, 5, 31-44. https://www.researchgate.net/publication/340849956_Impact_of_the_2019-20_coronavirus_pandemic_on_education

Oyeniran-Oluwashina, A., Oyeniran-Stella, T. , Oyeniyi, O., Ogundele, A., y Ojo, O. (2020). E-Learning: Advancement in Nigerian Pedagogy Amid Covid-19 Pandemic. International Journal of Multidisciplinary Sciences and Advanced Technology, 1(1), Special Issue Covid-19), 85-94. https://drive. google.com/file/d /1DeRGOouZAU9ir4QvqMJ_4Gs2V5grG00r/view

Özer, M. (2020). The Contribution of the Strengthened Capacity of Vocational Education and Training System in Turkey to the Fight against Covid-19. Journal of Higher Education, 10(2), 134-140. https:// doi.org/10.2399/yod.20.726951

Pan, H. (2020). A Glimpse of University Students' Family Life Amidst the COVID-19 Virus. Journal of Loss and Trauma. https://doi.org/10.1080/15325024.2020.1750194

Parrish, R., Stewart, M., y Duncan, S. (2020). Ophthalmologists Are More Than Eye Doctors-In Memoriam Li Wenliang. American Journal of Ophthalmology, 213, 1-2. https://doi.org/10.1016/j. ajo.2020.02.014

Peters, M., Wang, H., Ogunniran, M., Huang, Y., Verde, B., Chunga, J., Quainoo, E., Ren, Z., Hollings, S., Mou, C., Khomera, S., Zhang, H., Zhou, S., Laimeche, A., Zheng, W., Xu, R., Jackson, L. y Hayes, S. (2020, 8 de mayo). China's Internationalized Higher Education During Covid-19: Collective Student Autoethnography. Postdigital Science and Education. https://doi.org/10.1007/s42438-020-00128-1

Petersen, E., Hui, D., Hamer, D., Blumberg, L., Madoff, L., Pollack, M., Lee, S., McLellan, S., Memish, Z., Praharaj, I., Wasserman, S., Ntoumi, F., Azhar, E., Mchugh, T., Kock, R., Ippolito, G., Zumla, A., y Koopmans, M. (2020). Li Wenliang, a face to the frontline healthcare worker. The first doctor to notify the emergence of the SARS-CoV-2, (COVID-19), outbreak. International journal of infectious diseases, 93, 205-207. https://doi.org/10.1016/j.ijid.2020.02.052

Powell, K. (2016). Does it take too long to publish research? Nature, 530(7589), 148-151. https://doi. org/10.1038/530148a

Prem, K., Liu, Y., Russell, T., Kucharski, A., Eggo, R., Davies, N., Centre for the Mathematical Modelling of Infectious Diseases COVID-19 Working Group, Jit, M., y Klepac, P. (2020). The effect of control strategies to reduce social mixing on outcomes of the COVID-19 epidemic in Wuhan, China: a modelling study. The Lancet Public Health, 5(5), 261-270. https://doi.org/10.1016/S2468-2667(20)30073-6

Rodero, C., Díaz, A., Guerrero, M., y Duque, M. (2016). Análisis de la fiabilidad y validez de un cuestionario docente. ReVisión, 9(1), http://www.aenui.net/ojs/index.php?journal=revision\&page=article\& op=view\&path $\% 5 \mathrm{~B} \% 5 \mathrm{D}=219 \&$ path $\% 5 \mathrm{~B} \% 5 \mathrm{D}=373$

Rogero-García, J. (2020). La ficción de educar a distancia. Revista de Sociología de la Educación-RASE, 13(2), 174-182. http://doi.org/10.7203/RASE.13.2.17126. 
Satpathy, B., y Ali. E (2020). A study on psychological well-being of final year management students during COVID-19 pandemic lockdown in India. International Journal of Indian Psychology, 8(2), 1-25. http://ijip.in/articles/a-study-on-psychological-well-being-of-final-year-management-studentsduring-covid-19-pandemic-lockdown-in-india/

Shatakshi, L., y Nardev, S. (2020). CoVid-19: Unmasking the new face of Education. International Journal of Research in Pharmaceutical Sciences, 11(1), 48-53. https://doi.org/10.26452/ijrps.v11iSPL1.2122

Shenoy, V., Mahendra, S., y Vijay, N. (2020). Covid-19 Lockdown technology adaption, teaching, learning, students engagement and faculty experience. Mukt Shabd Journal, 9(4), 698-702. http://shabdbooks.com/gallery/78-april2020.pdf

Shimil, P. V., y Meenu, K. S. (2020). Morningness- eveningness and quality of night time sleep among adolescent students during COVID-19 lock-down period. International Journal of Indian Psychology, 8(2), 64-68. http://ijip.in/articles/morningness-eveningness-and-quality-of-night-time-sleepamong-adolescent-students-during-covid-19-lock-down-period/

Sintema, E. (2020). Effect of COVID-19 on the Performance of Grade 12 Students: Implications for STEM Education. EURASIA Journal of Mathematics. Science and Technology Education, 16(7), 1-6. https://doi.org/10.29333/ejmste/7893

Srivastava, A. (2020). Challenges faced by indian teachers and students in continuing education during institutional closure due to COVID-19 pandemic. A study. Gedrag \& Organisatie Review, 33(2), 697-704. https://doi.org/10.37896/GOR33.02/077

Sun, L., Tang, Y., y Zuo, W. (2020). Coronavirus pushes education online. Nature Materials, 19(6). https:// doi.org/10.1038/s41563-020-0678-8

Thomas, M. S. C., y Rogers, C. (2020, 25 de mayo). Education, the science of learning, and the COVID-19 crisis. Prospects, Comparative Journal of Curriculum, Learning, and Assessment/ Springer. https:// doi.org/10.1007/s11125-020-09468-z

Torres, J. (2020). Propuestas para el mejoramiento de la educación universitaria virtual después del brote del COVID-19. UVserva, 9(1), 4-7. https://doi.org/10.25009/uvserva.v0i9.2700

Torres, M., y Rovalo, M. (2015). Perfil de las Revistas Iberoamericanas sobre Educación Analizadas en el iresie. En J. Asenjo., O. Macías., y J. Toscano. (coord.), Congreso Iberoamericano de Ciencia, Tecnología, Innovación y Educación (pp. 1-20). OEI. https://www.oei.es/historico/congreso2014/memoriactei/1403.pdf

Trujillo, F., Fernández, M., Segura, A., y Jiménez, M. (2020). Escenarios de Evaluación en el contexto de la pandemia por la COVID-19: La opinión del profesorado. SantillanaLab. https://www.observatoriodelainfancia.es/ficherosoia/documentos/7074_d_SantillanaLAB.pdf

Van, W., y Parolin, Z. (2020). Covid-19, school closures, and child poverty: a social crisis in the making. The Lancet Public Health, 5(5), 243-244. https://doi.org/10.1016/S2468-2667(20)30084-0

World Health Organization [@WHO]. (2020, 11 de marzo). "This is not just a public health crisis, it is a crisis that will touch every sector - so every sector and every individual must be involved in the fight"- @DrTedros \#COVID19 \#coronavirus [Tweet]. Twitter. https://twitter.com/who/status/1237778490746945541

Yabin, H. (2020). Research on Online Education in the Midst of the COVID-19 Pandemic. Journal of Advances in Education Research, 5(2), 77-80. https://doi.org/10.22606/jaer.2020.52005 
- Revista de Ciencias Sociales y Humanidades. ISSN-P: 0188-9834 ISSN-E:2395-8669.

Zhang, Y., Geng, X., Tan, Y., Li, Q., Xu, C., Xu, J., Hao, L., Zeng, Z., Luo, X., Liu, F., y Wang, H. (2020). New understanding of the damage of SARS-CoV-2 infection outside the respiratory system. Biomedicine \& pharmacotherapy, 127, 1-7. https://doi.org/10.1016/j.biopha.2020.110195 

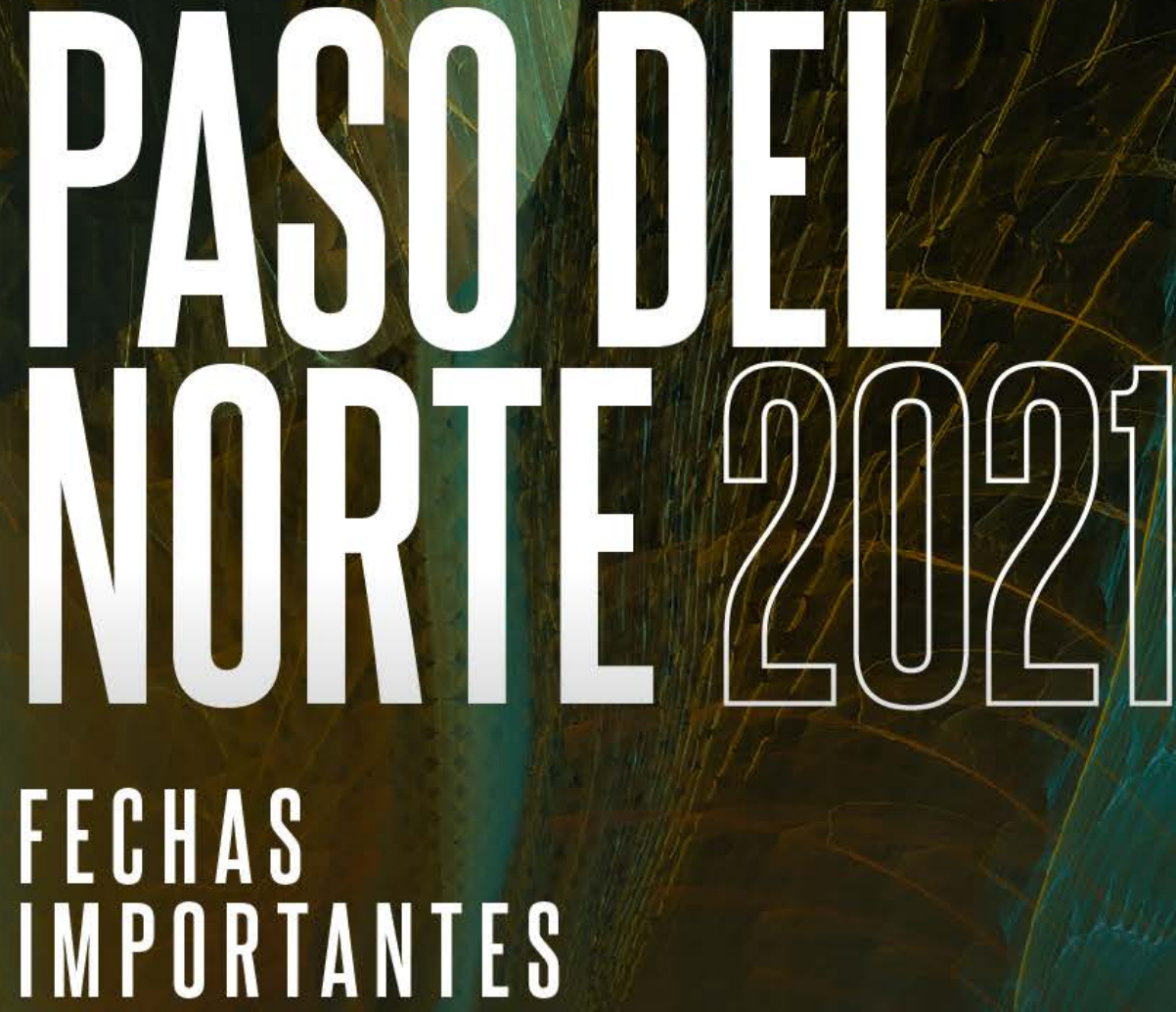

Recepción de trabajo

Del 15 de febrero al 7 de junio

Publicación de propuestas aceptadas 30 de julio

\begin{tabular}{ll}
\hline Fecha límite envío ponencias in extenso & $\mathbf{3 0}$ de septiembre \\
\hline Fecha límite de inscripción para ponentes & $\mathbf{8}$ de octubre \\
\hline Publicación del programa del Congreso & $\mathbf{2 2}$ de octubre \\
\hline Realización del Congreso & del $\mathbf{8}$ al $\mathbf{1 1}$ de noviembre \\
\hline Fecha límite para trámite de constancias & $\mathbf{3 0}$ de noviembre
\end{tabular}

Fecha límite para trámite de constancias

30 de noviembre

Registro de trabajos:

http://info.uacj.mx/congresocs2021 